\title{
Automatic Bell Pepper Colour Detector and Sorting Machine
}

\author{
Gophela Seiphepi, Adamu Murtala Zungeru, Jwaone Gaboitaolelwe, Caspar Lebekwe, Bokani Mtengi, \\ Department of Electrical, Computer and Telecommunications Engineering, Botswana International University of Science and \\ Technology, Private Bag 16, Palapye, Botswana.
}

ORCID: 0000-0003-2412-6559 (Adamu Murtala Zungeru)

\begin{abstract}
Sorting of products is a very difficult industrial process. Continuous manual sorting is tedious, time-consuming, and creates consistency issues. It is, therefore, admirable for companies to diversify into automation. Automation in the industrial workplace provides the advantages of improving productivity and quality while reducing errors and waste, increasing safety, and adding flexibility and efficiency to the manufacturing process. This paper presents the design and simulation of an automatic colour sensor and sorting machine (ACSSM) that automates grading and separating bell pepper according to colour. The system was designed for the food and packaging industry in Botswana, who experienced problems of spending enormous time and effort in sorting red, green, and yellow bell pepper according to colour after harvesting. Bell pepper is of high demand in all its ripening stages, and colour plays a crucial role in their pricing. Thus sorting is vital to meet the varying needs of customers and to obtain the most profits. The focal point was to design and test a circuit that would decrease the time, workspace, and effort while providing the essential function which was sorting of bell pepper. The implemented system is based on colour detection, object detection, and ejection to achieve sorting. The system performed well according to the design requirements when simulated on Proteus software.
\end{abstract}

Keywords: Sorting; Colour; Detection; Bell pepper; Conveyor belt

\section{INTRODUCTION}

Sorting of products is a very strenuous industrial process. Continuous manual sorting creates consistency issues and lowers efficiency in the workplace. Therefore, various automation techniques are being researched and applied to increase productivity, accuracy, and help eliminate human errors while improving workplace safety. In today's world of modern science and technology, the fundamentals of greater efficiency, quality, and automated machinery have greatly improved the industrial zone, the exponential growth of customer demands has led to the generation of industrial automation. Colour sensor systems are increasingly being used in automated applications to detect automation errors and monitor the quality of products at the production line's speed. They are used in assembly lines to identify and classify products by colour efficiently. Colour is the perceptual property of an object that appears to the observer when light rays are reflected from an object to their eyes.

Sir Isaac Newton discovered that white daylight consists of a spectrum of colours, namely red, orange, yellow, green, blue, and violet. He further found that only when light falls on an object that its characteristic colour is observed, and they are only three ways that an object interacts with light rays. When all the rays of light are absorbed, the human eye sees the colour black. When all are reflected, it sees white, and when all but one are absorbed, it sees the colour that is reflected. Similarly, when all the rays of light are absorbed for an electronic sensor, the sensor has zero input, which would be the colour black. When all are reflected, it senses an input of all 1's, which would be white, and when all but one are absorbed, it senses the colour that is reflected, which would be a combination of bits corresponding uniquely to each colour. The sensor's output will be the corresponding colour of the object, which is further used to facilitate automated sorting by an electronic circuit in an industrial workplace.

Colour is very critical in differentiating and sorting of products in industries, there are many other techniques of differentiating products which can be by weight, shape, and size. Colour is related to objects, materials, light sources, etc., based on their physical properties such as light absorption, reflection, or emission spectra. Different colour spaces help quantify color attributes numerically, for example, RGB colour space, which can be combined to form any color. A simple example is the red and green colour space shown in Fig. 1.

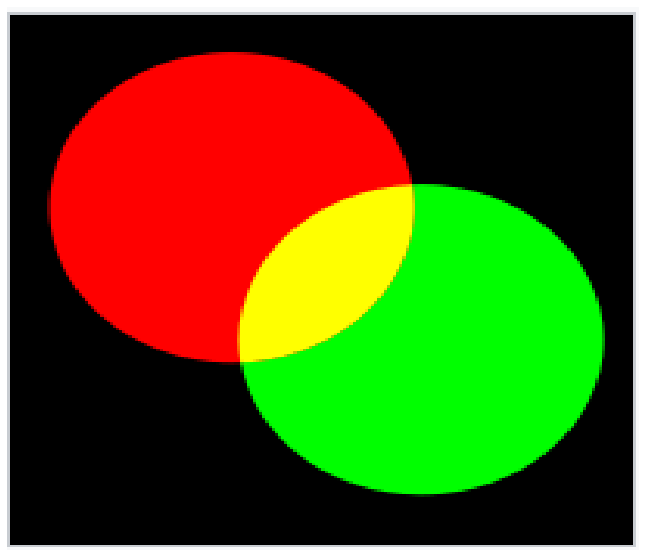

Figure 1: Red and green colour combinations or colour space [1]

Let there be three values which can be thought of as coordinates of a point in three-dimensional space, giving birth to the 
concept of colour space. Saturation, hue, luminance (HSL) is one such colour space. Hue is the particular degree of light or dark of a particular colour. Light intensity (lightness) measures the degree of whiteness or grayscale of a given colour, while saturation is a measure of the vividness of a given hue [2].

Recognizing various colours of objects is crucial in our day-today life to enhance our understanding of our environment and interaction with it. This project focuses on achieving artificial colour vision using the application of electronics. The perception of colour is derived from the wavelength and intensity of light in the visible spectrum. Colour detection has a variety of applications in industries to facilitate the production and packaging process. In food industries, the colour of products can be used as a quality control measure while in others such as automobiles, paint industries, and textile products, input materials can be sorted according to their colours with the assistance of colour sensor systems. In addition, during the bottling of products, colour detection can be used to inspect bottles with a bottle cap from those that do not have. They are also applied to sorting fruits and vegetables, where objects to be sorted are scanned with a colour video camera. The signals from the camera are digitalized and recognize the different coloured fruits and vegetables. In the system, the camera's collected data is passed to the colour sorter processor to finalize the good or bad fruits or vegetables [3].

Similarly, another way of sorting consists of a colour sensor, servo motors, and microcontroller, which synchronizes a robotic arm's movement to pick the objects moving on a conveyor. It aims to sort the coloured objects on the conveyor belt by picking and placing them in their respective preprogrammed batches. Thereby eliminating the monotonous work done by humans and achieving accuracy and speed in the workplace [4].

In this project, colour detection is carried out using light dependent resistors (LDR's) and light sources. The light source is used to improve the reflected light rays from an object, whereas the LDRs (light dependent resistors) are used to sense the intensity of the reflected light from the object and vary their resistances accordingly, as shown in Fig. 2. Similarly, Fig. 3 illustrates the various light intensities of visible light against frequency $(\mathrm{nm})$.

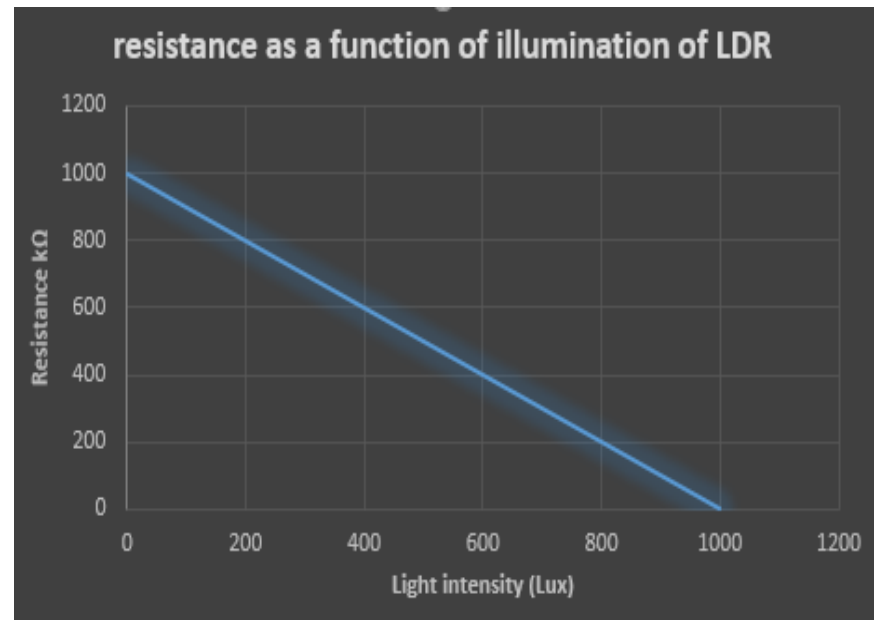

Figure 2: Resistance as a function of illumination

\section{Relative Intensity of Visible Light vs Frequency}

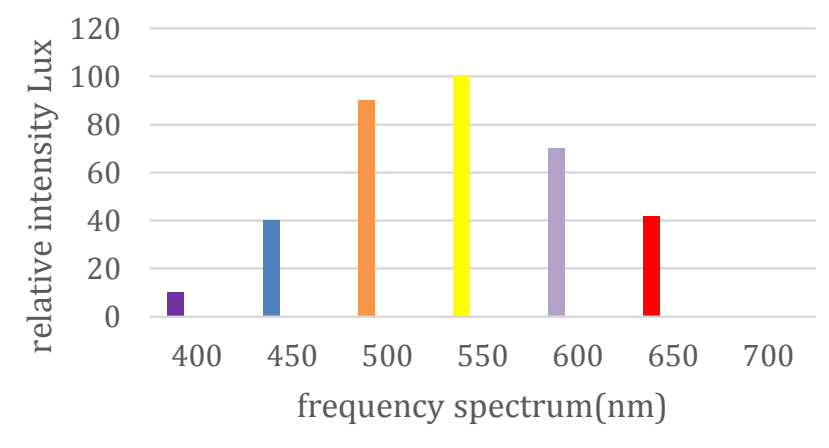

Figure 3: Relative intensity of light vs frequency

This research aimed to design, implement, and test an automatic colour sorting machine that would decrease the time, workspace, and effort while providing the basic function of sorting products in an industrial workplace. The system was intended to autonomously detect and sort bell peppers according to colour to mitigate the problem of low efficiency and sorting errors in a bell pepper industrial processing and packaging company in Botswana. This paper's remainder illuminates the functionality of the automatic colour sensor and sorting machine (ACSSM).

\section{MATERIALS AND METHODS}

This section provides details on the proposed system. The system comprises four subsystems: power supply, object detector, colour sensor, and ejection controller, as shown on the block diagram in Fig 4.

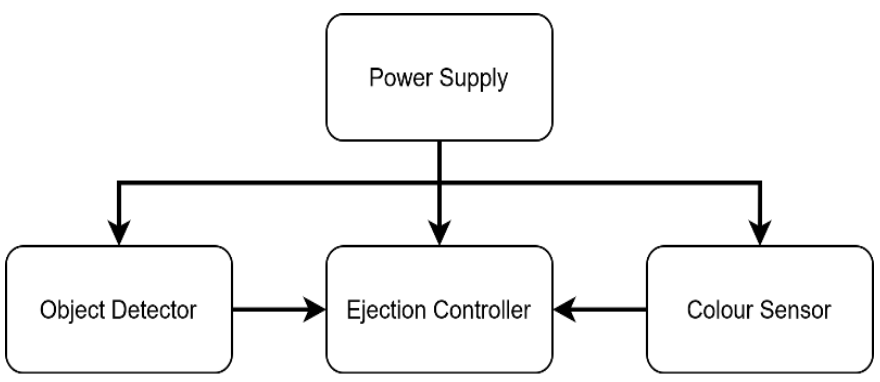

Figure 4: Block diagram showing major parts of the ACSSM

\section{Power Supply}

The power supply provides 12 volts DC sufficient to power the different subsystems from a $220 \mathrm{~V}$ AC source, which is the standard voltage per home in Botswana, similar to those used in [5-7]. A transformer steps down the $220 \mathrm{~V} \mathrm{AC}$ before it is further converted to dc voltage using a bridge rectifier. The $12 \mathrm{~V}$ DC output is further filtered using capacitors to remove noise or unwanted frequencies to produce a pure 12-volt output signal of $0 \mathrm{~Hz}$, as shown in Fig. 6. 


\section{Calculations for the capacitor.}

Taking AC voltage at the primary coil as $220 \mathrm{~V}$ and voltage at the secondary coil was $22.0 \mathrm{~V}$

$$
\begin{aligned}
\left(N_{P} / N_{S}\right) & =\left(V_{P} / V_{S}\right)=\left(\frac{I_{P}}{I_{S}}\right) \\
& =220 / 22=10.0 \\
\frac{220}{22}: \frac{22}{22} & =11: 1
\end{aligned}
$$

Hence ratio of transformer $=11: 1$

$$
\begin{aligned}
& V r m s=V s=\frac{220}{22} \\
& V p=12 * \sqrt{2}=17 \mathrm{~V}
\end{aligned}
$$

Assuming a ripple voltage of $20 \%$

$$
\begin{aligned}
& d v=\left(\frac{20}{100}\right) * 17=3.4 \mathrm{~V} \\
& d f=\frac{1}{2 f}=\frac{1}{100}=0.01 \\
& C 1=1 * \frac{0.01}{3.4}=0.003571428=357 \mu F
\end{aligned}
$$

\section{Object Detector System}

The object sensing system is used to power a conveyor belt run by a $12 \mathrm{~V}$ dc motor as shown in Fig. 7 . The system turns the conveyor motor on when there is no bell pepper present at the colour sensor and turns the conveyor motor off only when a bell pepper is ready for colour sensing. It performs this function with the application of an LDR and a light source. When a bell pepper obstructs the light source from shining onto the LDR the system cuts off power to the conveyor belt motor, which signals the presence of a bell pepper ready for colour sensing. When there is no bell pepper to obstruct the light source from shining onto the LDR, the system continues to run the conveyor motor until a bell pepper obstructs the light source from shining onto the LDR.

\section{Colour Sensor}

The detection principle embedded in the colour sensor system is based on the reflectance property of a coloured surface. When white light is shone on a surface, the surface will reflect a specific spectrum while absorbing all the other spectra. For example, a white light focused on a red surface is reflected as red. The reflected red light impinges on the light transducer producing a corresponding output voltage that is typical of the reflected red colour. By interpreting this output voltage using an interface circuit, the colour of bell peppers can be determined.

Light-dependent resistors (LDR) are used in the proposed colour sensor system as the principal detector element. LDR's generate electron and hole carriers upon exposure from visible light inducing photo-current. The maximum photo-current can be given by;

$$
I_{L D R}=(1-R)\left(1-e^{a d}\right) q\left(\frac{P_{i n}}{h V}\right)
$$

Where $\mathrm{R}$ is the surface reflectivity of the LDR, $\mathrm{d}$ is the depth from the silicon surface, $\alpha$ is the absorption coefficient, dependent on the material property (e.g., silicon or GaAs) $q$ is the electron charge. $P_{\text {in }}$ is the power of incident light; $h$ is Planck's constant; and $\mathrm{v}$ is the frequency of incident light [8].

The colour sensing sub-circuit detects the bell pepper's color by converting the RG colour space (red and green) into digital outputs. The blue colour space is not used since it is not important in the system's overall function. Hence two LDR's are used, one responsible for red colour detection shown in Fig. 8 and the other for green colour detection shown in Fig. 9. When the red light intensity reflected from red bell pepper is shined onto the LDR responsible for red colour detection, a corresponding transistor is turned on, which is converted to a digital 1. If any other colour is reflected onto the red detecting LDR the transistor will remain in a low state(off), similarly for the green detecting LDR. This is possible since only colours in the RG colour space is used.

\section{Ejection Controller}

The bell pepper ejection controller consists of an AND gate based decoder used to drive relay controlled de motors which produce a linear motion with the use of a rack and pinion to eject the bell pepper to the right location after colour sensing. The truth table shown in Table. 1 is used by the decoder to determine the right ejection motor which places the bell pepper in the right batch to achieve sorting of the bell peppers. Ejection motors with rack and pinions are placed at different angles to eject the various coloured bell peppers into their respective batches. The reset spring restores the rack to the original position after a bell pepper is sorted. It can easily restore the rack since logic gates will disable the motor once a bell pepper is ejected. The object detector simultaneously signals the conveyor to feed another bell pepper for evaluation, which is similarly ejected according to colour by the corresponding rack and pinion motor. The rack and pinion motor setup continuously eject and reset to perform iterations of sorting. Fig. 5 and Fig. 10 show the ejection motor setup and the Ejection controller circuit, respectively.

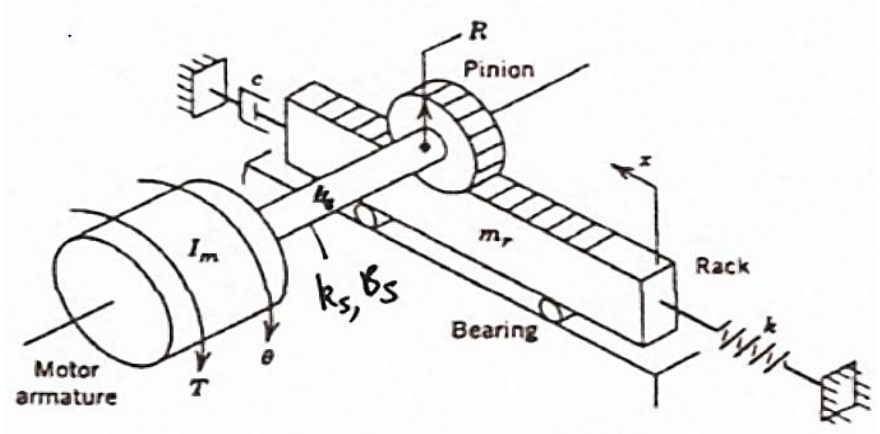

Figure 5: Ejection motor with reset spring and rack and pinion [9] 
Table 1: 2 to 4 decoder output.

\begin{tabular}{|c|c|c|c|c|c|c|}
\hline Enable & Red signal & Green signal & $\begin{array}{c}\mathrm{Y}_{3} \\
\text { Yellow }\end{array}$ & $\begin{array}{c}\mathrm{Y}_{2} \\
\text { Red }\end{array}$ & $\begin{array}{c}\mathrm{Y}_{1} \\
\text { Green }\end{array}$ & $\begin{array}{c}\mathrm{Y}_{0} \\
\text { Reject }\end{array}$ \\
\hline 0 & 0 & 0 & 0 & 0 & 0 & 0 \\
\hline 0 & 0 & 1 & 0 & 0 & 0 & 0 \\
\hline 0 & 1 & 0 & 0 & 0 & 0 & 0 \\
\hline 0 & 1 & 1 & 0 & 0 & 0 & 0 \\
\hline 1 & 0 & 0 & 0 & 0 & 0 & 1 \\
\hline 1 & 0 & 1 & 0 & 0 & 1 & 0 \\
\hline 1 & 1 & 0 & 0 & 1 & 0 & 0 \\
\hline
\end{tabular}

\section{V DC Power Supply}

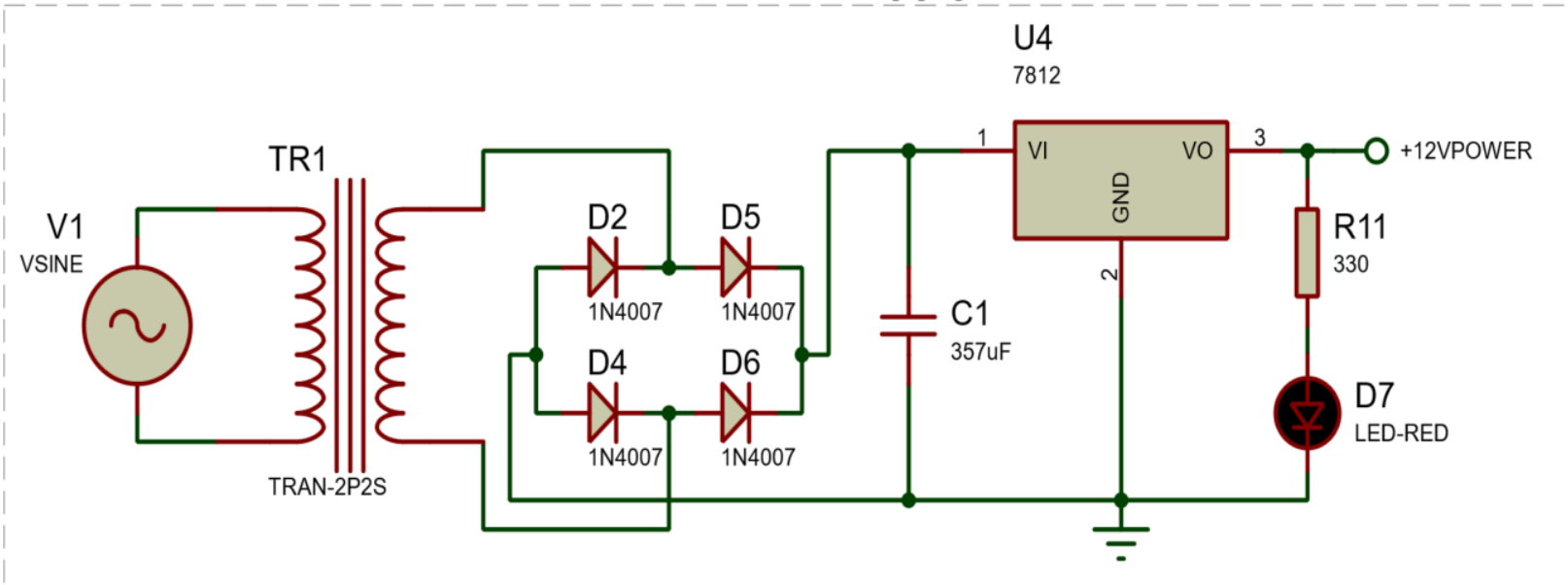

Figure 6: 12V DC Power Supply

\section{Object Detector}

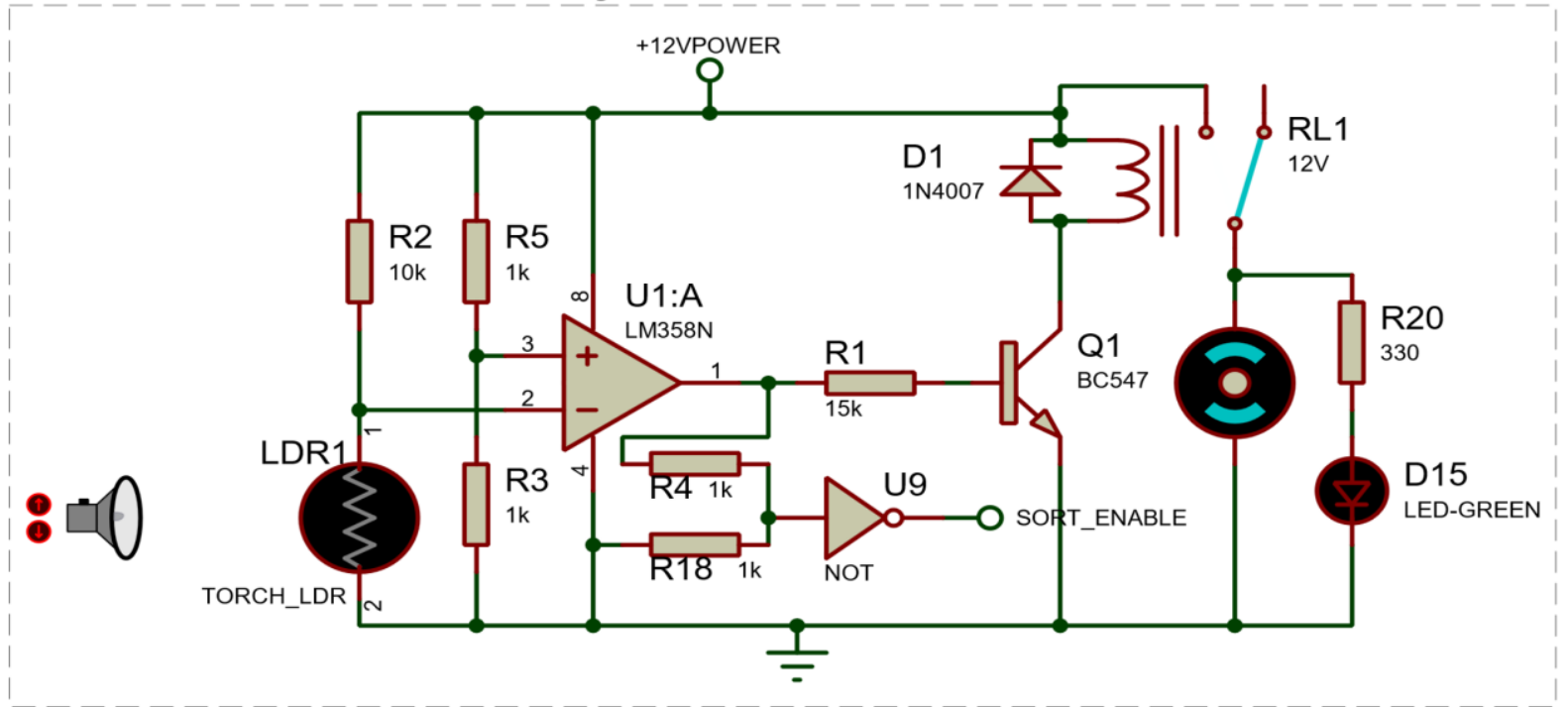

Figure 7: Bell Pepper Detector on a conveyor belt 


\section{Red Colour Sensor}

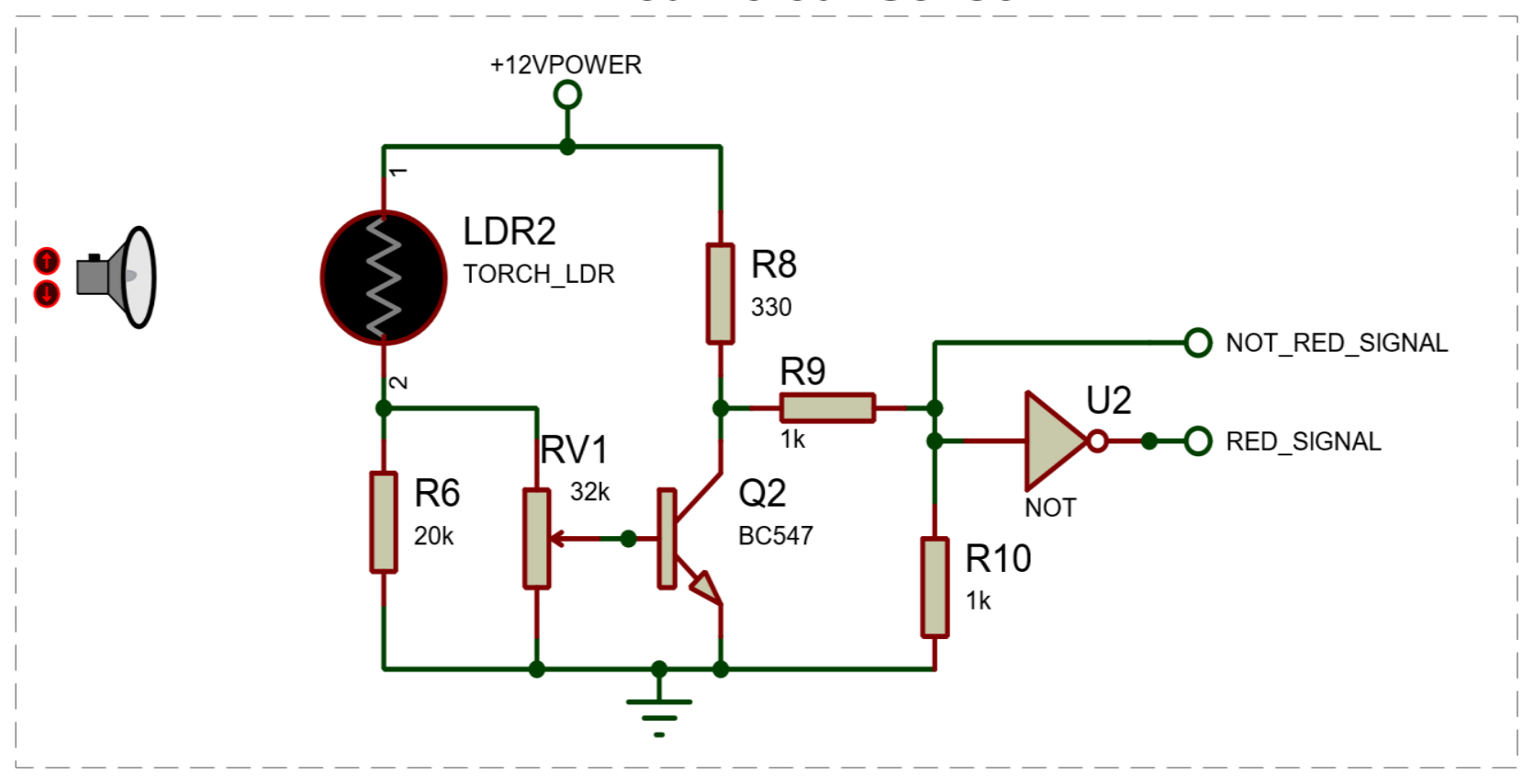

Figure 8: Red pepper detector colour sensor

\section{Green Colour Sensor}

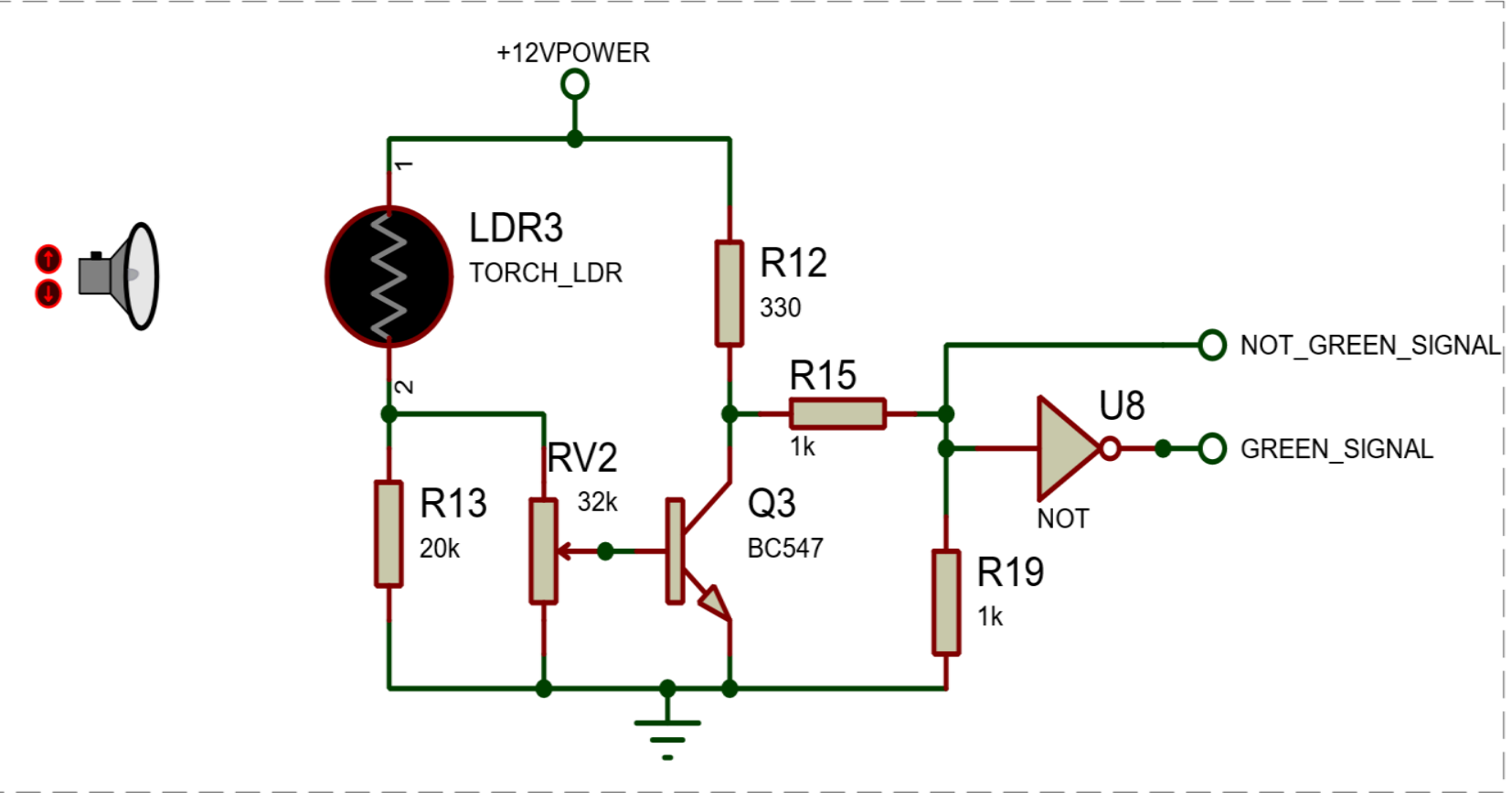

Figure 9: Green pepper detector colour Sensor 


\section{Object Ejector}

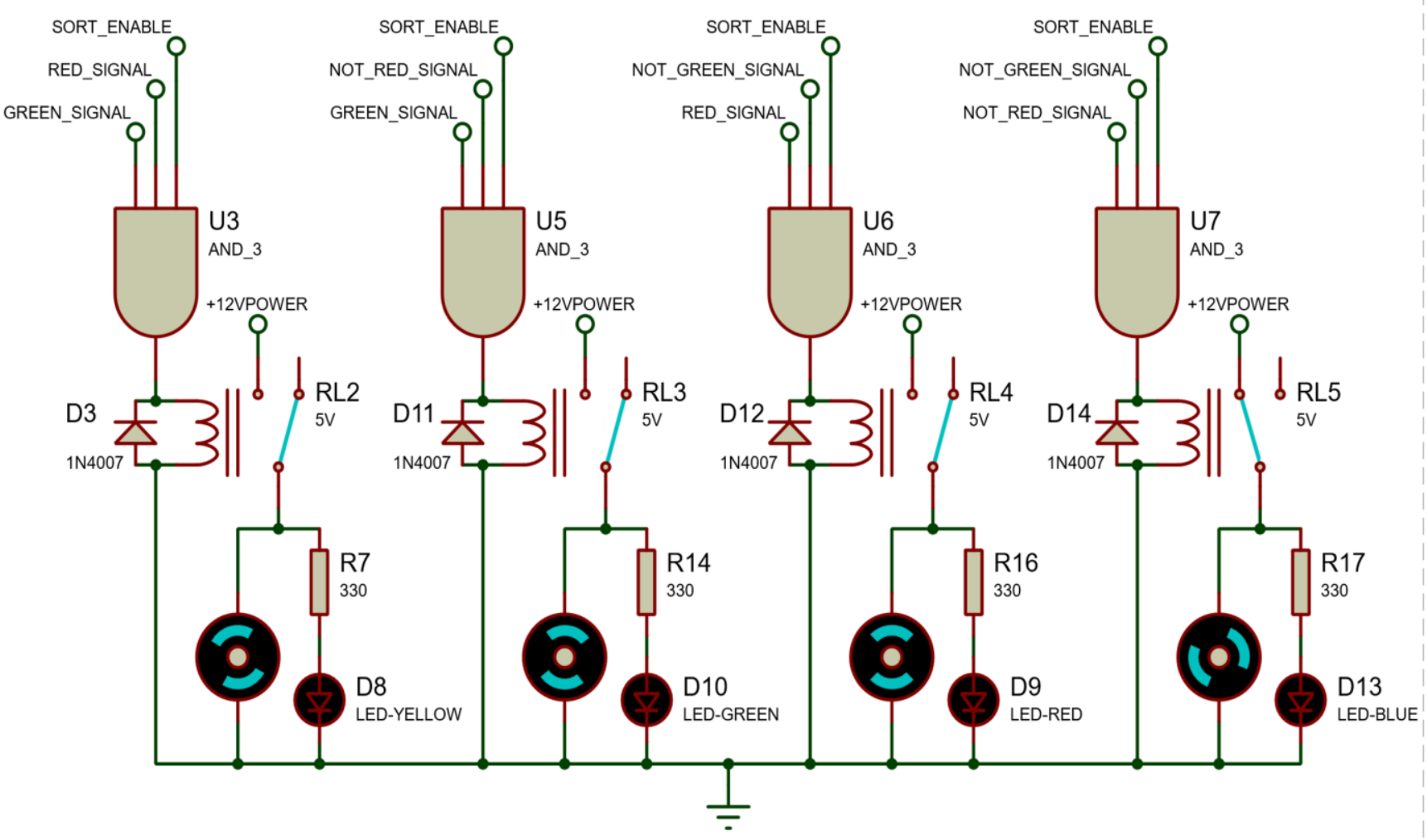

Figure 10: Object Ejection Controller

\section{RESULTS AND DISCUSSION}

I. Power Supply

The power supply gave an output of $12 \mathrm{v}$ when connected to the $220 \mathrm{~V}$ supply. It managed to power all subsystems without fail, which was one of the requirements. This requirement was tested by combining all the subsystems and powering them during the testing of bell peppers. Fig. 11 shows the power supply system.

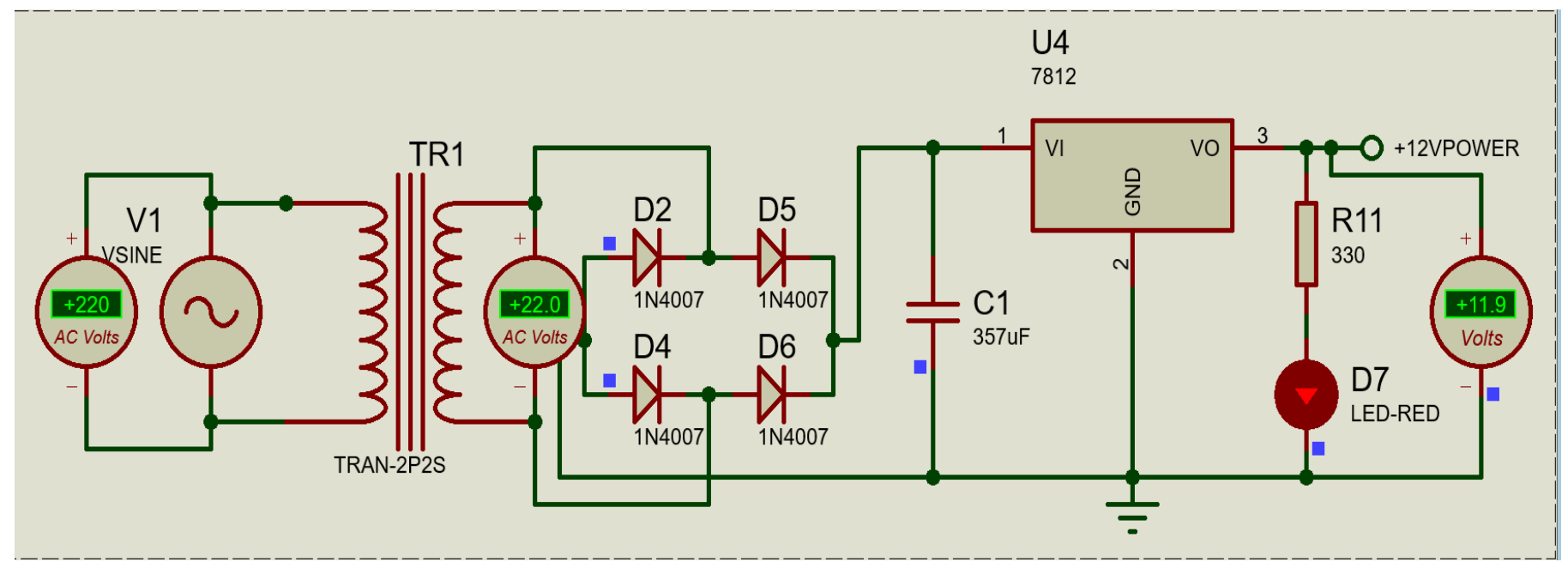

Figure 11: Power Supply

II. Object Detection System

The object detection system was required to operate in two cases. Firstly, when there is no bell pepper in front of the object detector LDR. Secondly, when there is a bell pepper in front of the LDR. 


\section{i. $\quad$ Case 1-No Bell Pepper}

When there was no bell pepper in front of the LDR, light from the light source reached the LDR of the object detector, thus leading to the LDR resistance to decrease. Consequently, the non-inverting terminal voltage became higher than the inverting terminal, thus leading to the Op-amp output voltage of $10.44 \mathrm{~V}$. The OP-amp output was enough to switch on the transistor and the relay, hence turning on the conveyor belt motor to feed in bell peppers. This is shown in Fig. 12.

\section{ii. $\quad$ Case 2- Bell Pepper Present}

When a bell pepper was in front of the LDR, light from the light source to the LDR was blocked by incoming bell pepper, and the LDR resistance increased, the non-inverting terminal voltage became lower than the inverting terminal. The Op-amp output was thus triggered to $0 \mathrm{~V}$. The transistor and relay turned off, and supply to the conveyor motor was cut off, and the belt stopped running as shown in Fig 13, thus stopping a bell pepper in the position of the colour sensing system.

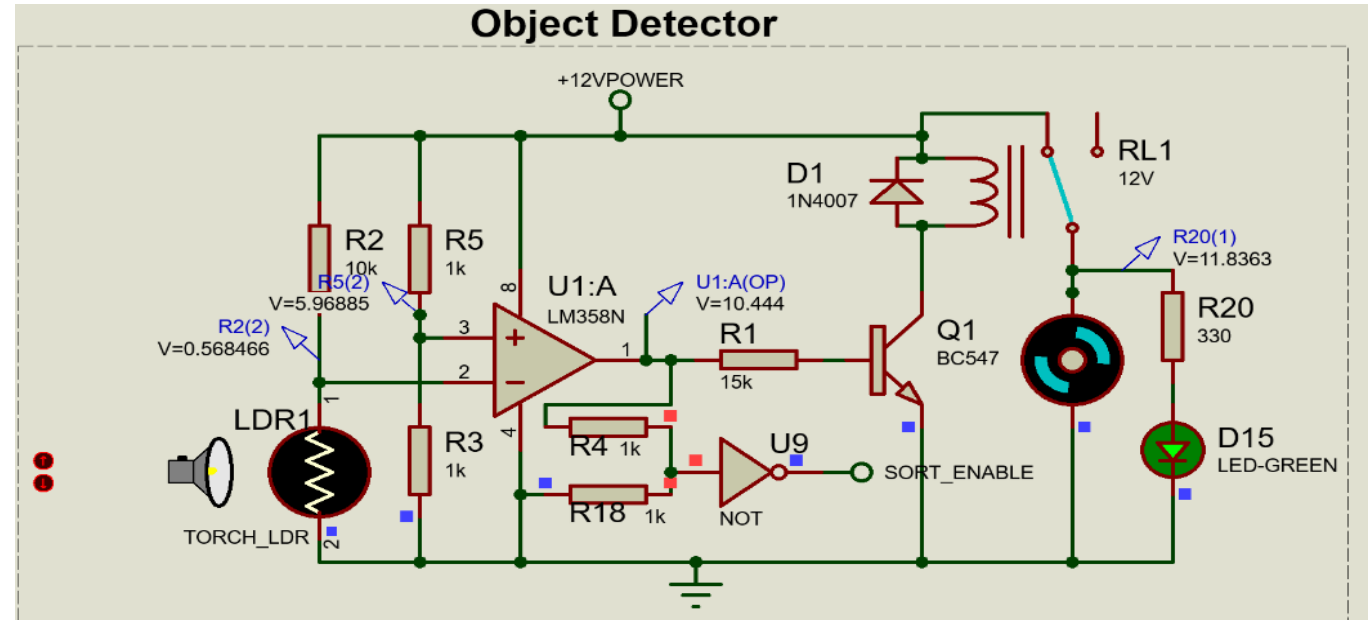

Figure 12: Object Detector in case 1

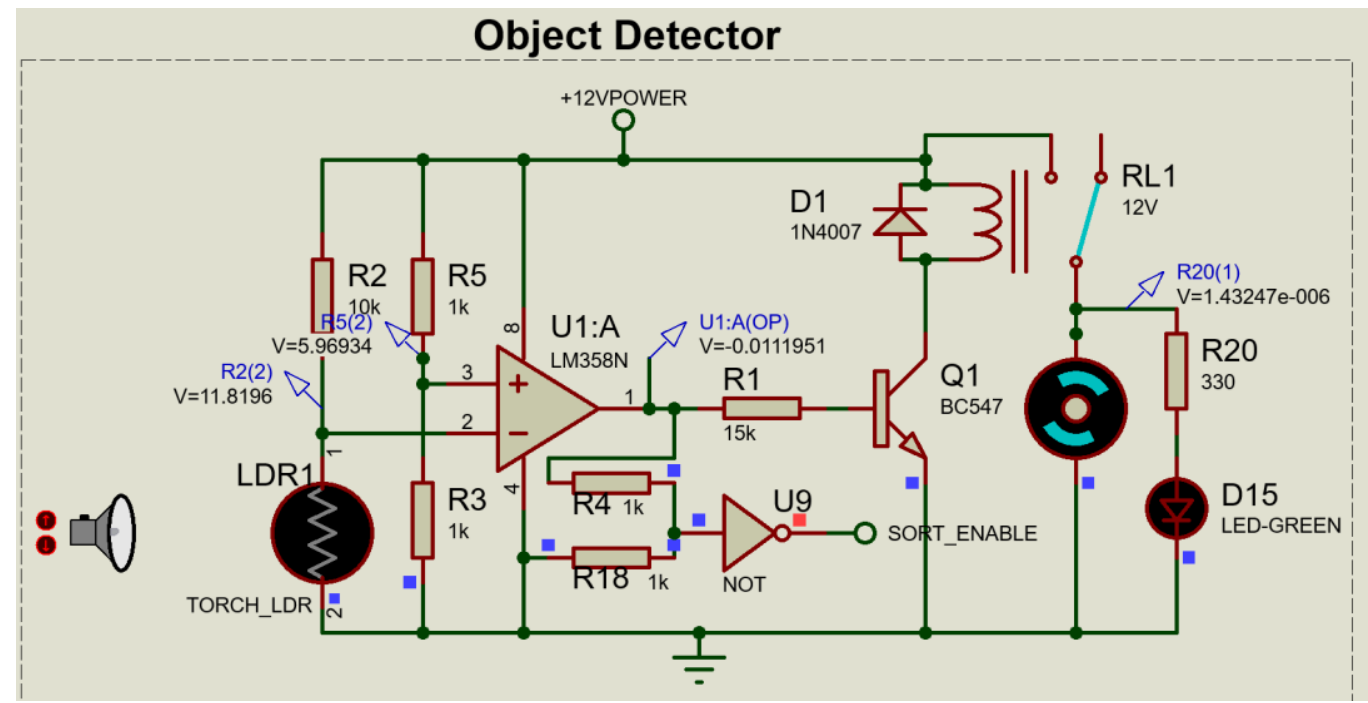

Figure 13: Object Detector in case 2

\section{Colour Sensing}

The colour sensor system was required to accurately distinguish the various coloured bell peppers. Variable resistors RV1 and RV2 of the red and green colour sensors circuits shown in Fig. 8 and Fig.9 were calibrated through trial and error to find the right resistance to bias the transistors Q2 and Q3 to turn on at red and green light intensity respectively. It was ensured that the base-emitter voltages of the transistors were roughly above $0.7 \mathrm{~V}$ at the presence of the required light intensities for the transistors to operate as switches. Fig.14, Fig. 15, Fig. 16, and Fig.17 show the results of the colour sensor tests. 


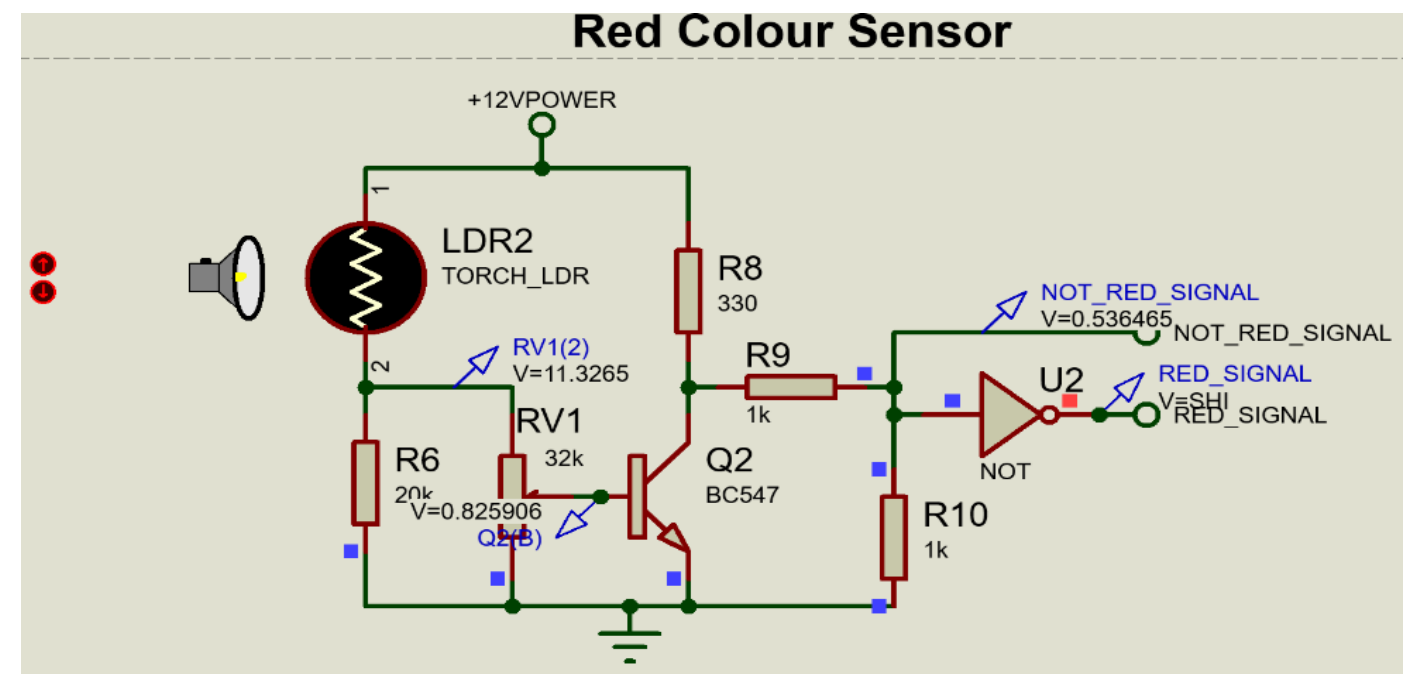

Figure 14: Red Colour Detection

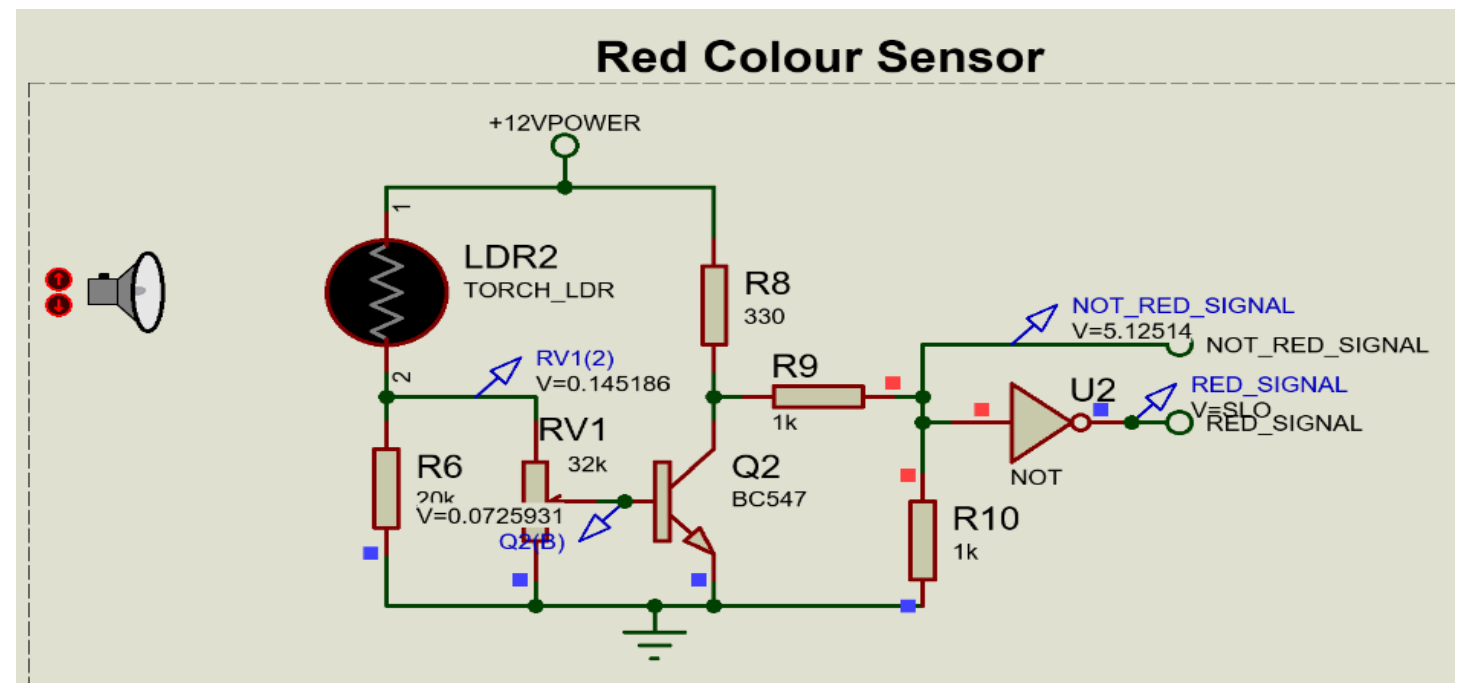

Figure 15: Absence of Red colour at Red colour sensor

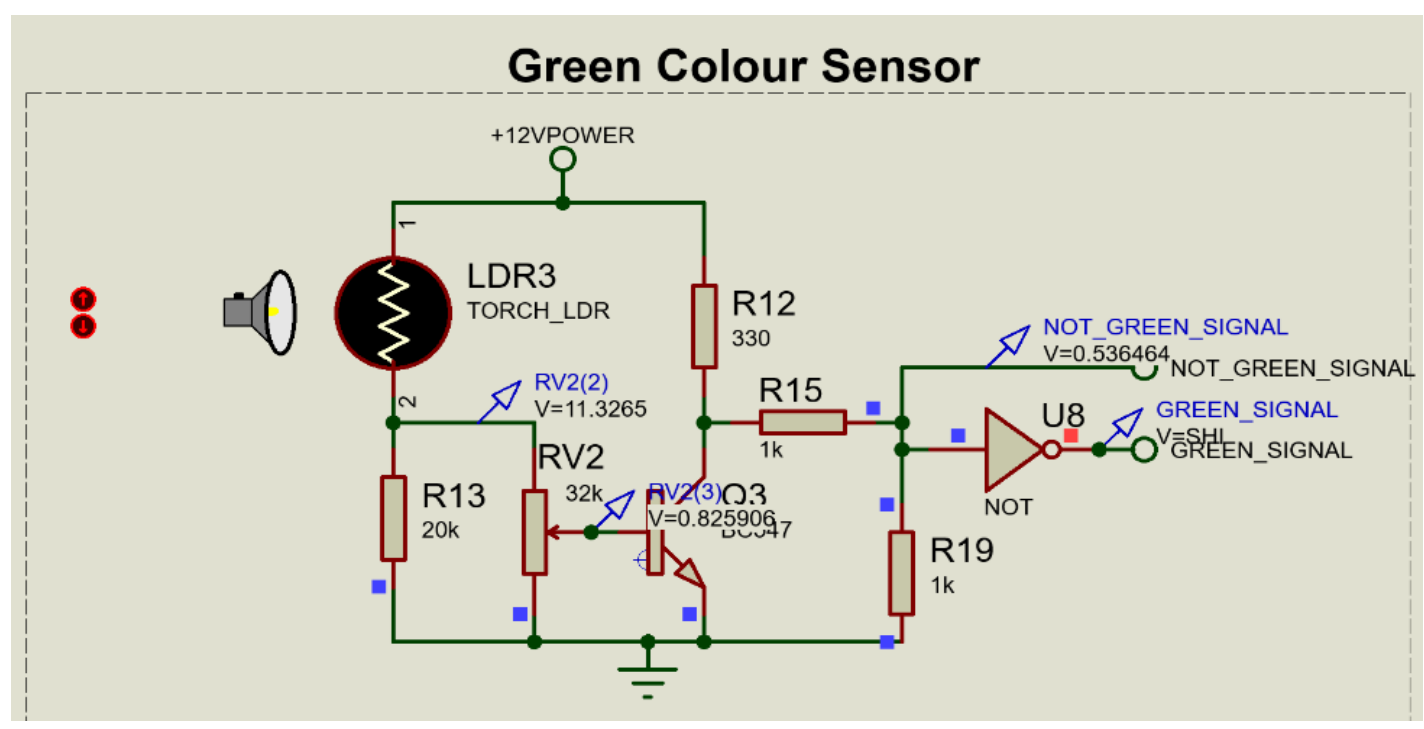

Figure 16: Green colour detection 


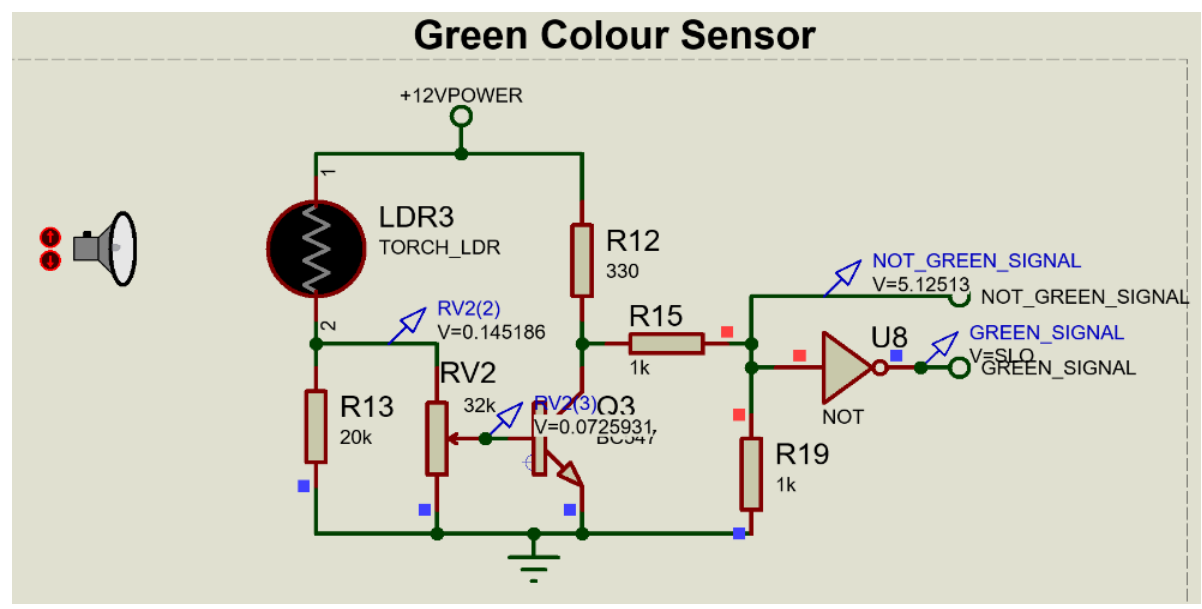

Figure 17: Absence of green colour at Green colour sensor

\section{Bell Pepper Ejection}

The ejection system was required to eject the bell peppers according to their colour. The decoder used signals from the colour sensors and object detectors to select the ejection motor, which would place the bell pepper in the right batch according to its colour. The ejection motor's angle and position were adjusted to meet the bell peppers' requirement for accurate sorting into their respective batches.

Tests of the bell pepper Ejection controller;

\section{i. $\quad$ Case 1: Yellow pepper testing}

Enough yellow light was reflected from the tested pepper; thus, a sufficient voltage drop fell across both LDR's enough to turn both transistors Q2 and Q3 on since yellow is a combination of both red and green light intensity. Consequently, this led to the decoder selecting and switching on the yellow pepper ejection motor, as shown in Fig. 18.

\section{ii. $\quad$ Case 2: Reject Bell pepper}

Not enough light intensity was reflected from the tested object, thus very little or no voltage drop fell across both LDR's. Both transistors Q2 and Q3 remained off, hence resulting in the decoder selecting and switching on the reject pepper ejection motor, as shown in Fig. 19.

\section{iii. $\quad$ Case 3: Green pepper testing}

Enough, green light intensity was reflected from green pepper; hence a sufficient voltage drop fell across LDR 2 enough to turn transistor Q3 on while transistor Q2 remained off. Consequently, this led to the decoder selecting and switching on the green pepper ejection motor as shown in Fig. 20.

\section{iv. Case 4: Red pepper testing}

Enough red light was reflected from the red pepper. Hence a sufficient voltage drop fell across LDR 1 enough to turn transistor Q2 on while transistor Q3 remained off. Consequently, this led to the decoder selecting and switching on the red pepper ejection motor, as shown in Fig. 21.

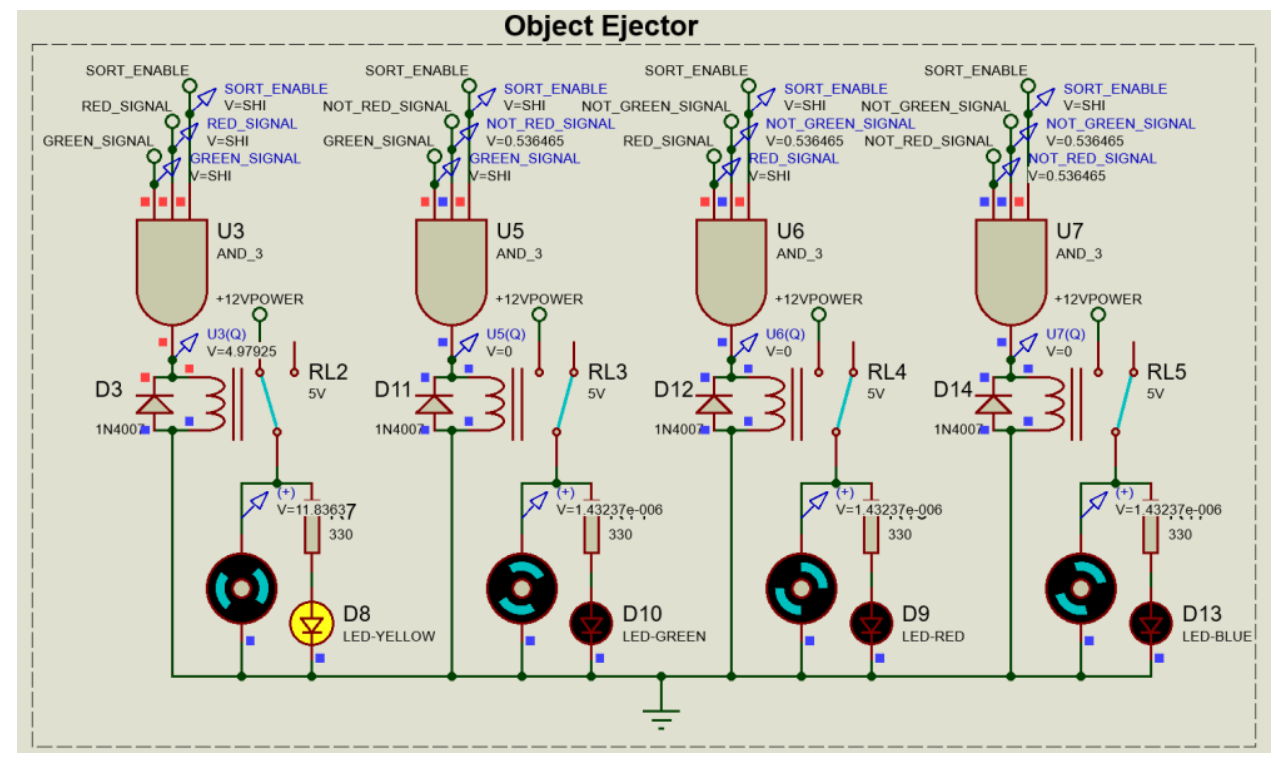

Figure 18: Yellow pepper test 
International Journal of Engineering Research and Technology. ISSN 0974-3154, Volume 13, Number 11 (2020), pp. $3156-3166$ (C) International Research Publication House. https://dx.doi.org/10.37624/IJERT/13.11.2020.3156-3166

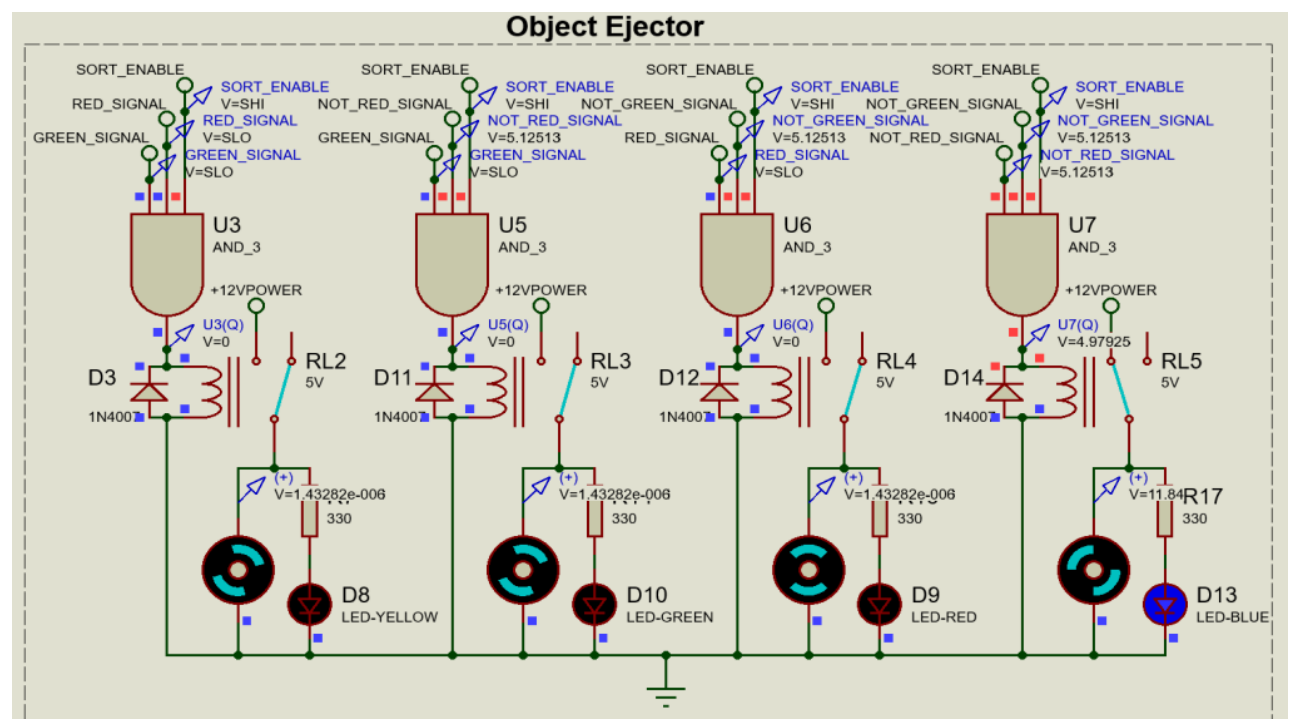

Figure 19: Reject or unwanted pepper test

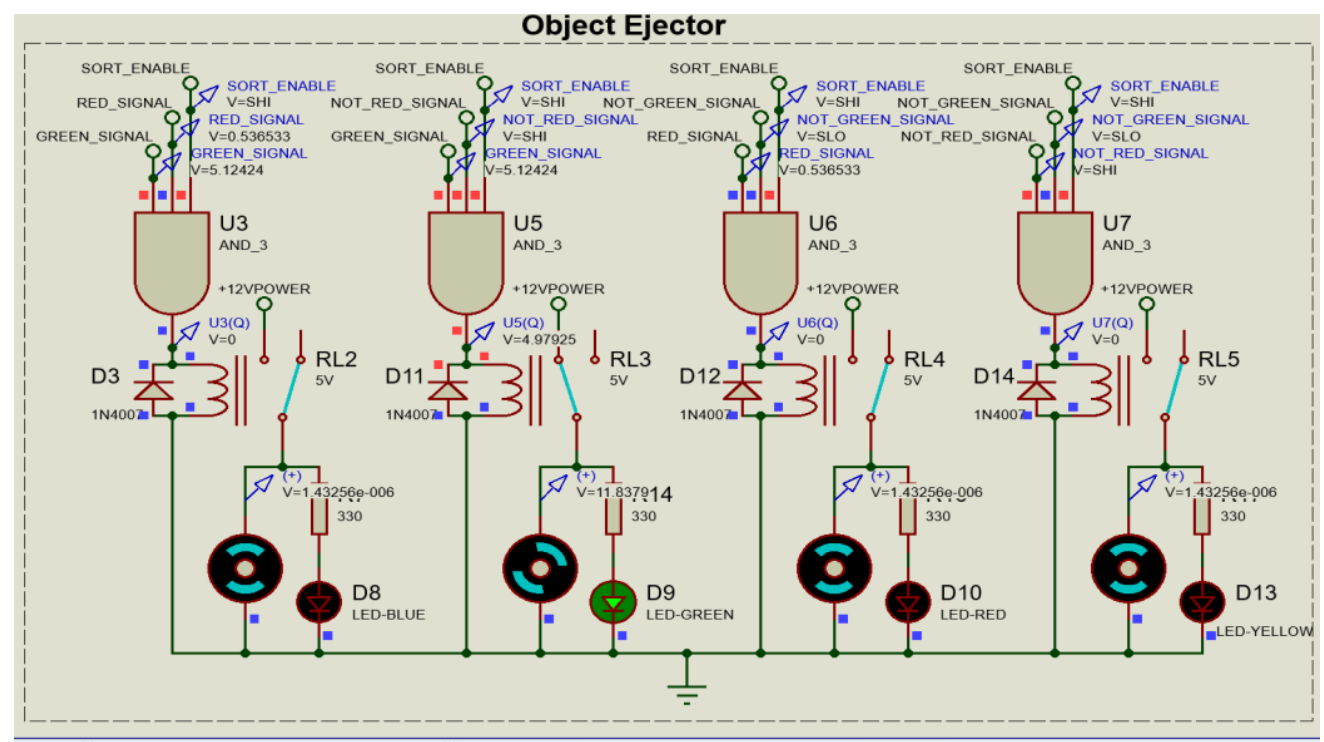

Figure 20: Green pepper test

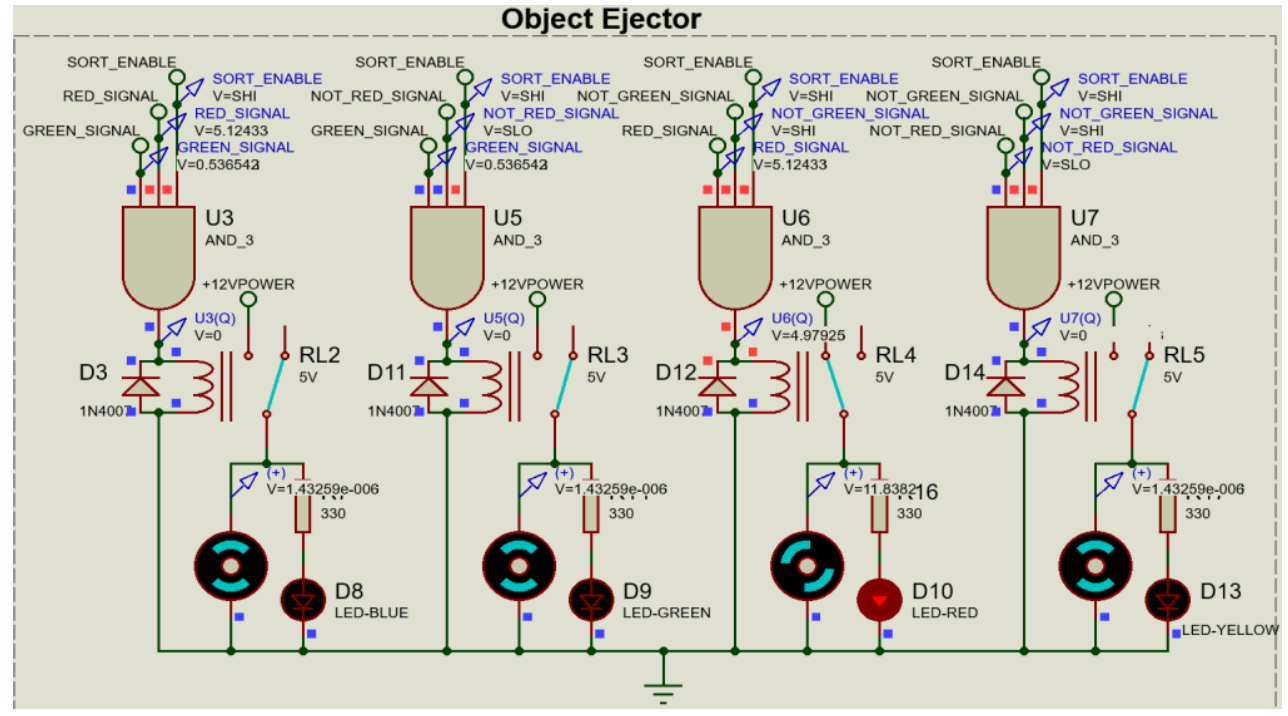

Figure 21: Red pepper test 


\section{CONCLUSION}

In this work, the design, simulation, and analysis of the Automatic Colour Sensor and Sorting Machine (ACSSM) were presented. The main aim of the work was to design a circuit that would decrease the time, workspace, and effort while providing the basic function, which was sorting of bell peppers by colour. The system was designed and simulated in Proteus. Based on the results, the system has achieved its aim. The ACSSM was able to repetitively detect the colour of bell peppers and sort them according to colour. The simulations demonstrated the ability to sense the bell peppers' presence and color and sort them by driving the correct motor to eject the bell pepper to the right batch according to its colour. The project can therefore be remodeled to solving other problems that require colour sorting.

\section{REFERENCES}

[1] D.Yerrick," Illustration of the additive RG colour space: two overlapping red and green circles," 2009

[2] A. Deshmukh,M, Nagane and V. Awatade," Design \& Development of Automatic Conveyor Controlling System for Sorting of Component on Colour basis," Vol.5, No.2, p.1862,2016

[3] R.Mahendran, GC. Jayashree and K. Alagusundaram," Application of Computer Vision Technique on Sorting and Grading of Fruits and Vegetables" 2011

[4] D.Banupriya, B.Nivedhitha and T.Priyanka," Design And Development Of Vision Sorting Machine," in Pryiadarshini Engineering Collage,2019

[5] M. Assaad, I. Yohannes, A.Bermak, D. Ginhac and F. Meriaudeau," Design and Characterization of Automated Colour Sensors System"

[6] A.M. Zungeru, J. Gaboitaolelwe, B. Diarra, J.M. Chuma, L. M. Ang, L. Kolobe, M. David, I. Zibani, "A Secured Smart Home Switching System based on Wireless Communications and Self -Energy Harvesting", in IEEE Access, vol. 7, pp. 25063 25085, 2019. DOI: https://doi.org/10.1109/ACCESS.2019.2900305

[7] B. Diarra, A.M. Zungeru, S. Ravi, J.M. Chuma, B. Basutli, I. Zibani, "Design of a Photovoltaic System with Ultracapacitor Energy Buffer," Procedia Manufacturing (Elsevier), 33(2019), pp. 216-223, 2019.

[8] A.M. Zungeru, M. Mangwala, J.M. Chuma, B. Gaebolae, B. Basutli, "Design and Simulation of an Automatic Room Heater Control System", Heliyon, Elsevier, vol. 4, Issue 6, Article number e00457, 2018.

DOI: https://doi.org/10.1016/j.heliyon.2018.e00655

[9] D. Marano, F. Pellicano, E. Pallara, A. Piantoni, L. Tabaglio, M. Lucchi and, S. Orlandi, "Modelling and simulation of rack-pinion steering systems with manufacturing errors for performance prediction", International Journal of Vehicle Systems Modelling and Testing. Vol. 13, No. 2, pp. 178-198, 2018. 